(C) Journal of Applied Mathematics \& Decision Sciences, 1(2), 81-87 (1997)

Reprints Available directly from the Editor. Printed in New Zealand.

\title{
Limit Policies in N-Sector Dynamic Growth Games with Externalities
}

\author{
RONALD D. FISCHER \\ rfischer@dii.uchile.cl \\ Centro de Economía Aplicada, Depto. Ingeniería Industrial, Universidad de Chile \\ LEONARD J. MIRMAN \\ lm8h@fermi.clas.virginia.edu \\ Dept. of Economics, U. of Virginia, Charlottesville, VA 19104
}

\begin{abstract}
We examine an economy with $n$ production sectors that interact via a production externality. We find a solution to the resulting dynamic differential game between sectors and compare it to the cooperative solution. As the number of sectors increases, the limiting policy is the optimal policy without a production externality. This policy is inefficient and, depending on the sign of the externality between sectors, the inefficiency is due to over- (or under-) consumption.
\end{abstract}

Keywords: Dynamic game, growth, externalities.

\section{Introduction}

The object of this paper is to examine the dynamics of growth and the optimal investment policies in a model with several sectors, where an externality is partially internalized, in contrast to the usual assumption of no internalization. In our model of an economy, the output of each sector influences the output of the other sectors. As an example, external effects due to spillovers from education or innovation in one sector have a positive effect on the other sectors of the economy. On the other hand, there could be negative external effects due to, say, pollution. Each sector in the model is assumed to maximize its own utility. We assume that the effect of the output of other sectors cannot be altered by the actions of the affected sector since output decisions are taken noncooperatively. Hence, this model is studied in the context of a differential game in which the externalities are only partially internalized be each of the sectors. The Nash equilibrium of the game is therefore inefficient.

Our first task is to reformulate the model of Fischer and Mirman [3], in which the original differential game with this type of externality is analyzed, as a model of the choice of sectoral-specific labor input in an economy with $n$ sectors. This formulation is more general than the usual dynamic economic model and it is consistent with the models used in the literature on endogenous growth (Romer [1]). We then specialize the model so that the production function is Cobb-Douglas and the objective function is logarithmic. This is done in order to make the model tractable while retaining the most important features of the general model. In fact, we are able to get closed form solutions to the resulting differential game between 
sectors. It is then easy to make comparisons between the cooperative and the noncooperative solutions.

This formulation also allows us to study other questions. In particular, we study the convergence of the noncooperative solution as the number of sectors increases. In this way we can study the effect of the externality on the optimal (noncooperative) policy of each sector. The major result of the paper is to show that, as the number of sectors grows, so that the influence of any specific sector plays an increasingly smaller role on the other sectors, the noncooperative policy converges. The limiting policy is the optimal cooperative policy in the fishing model of Levhari and Mirman [4], where there are no production externalities. This limiting policy is not, however, the optimal policy, which would have to account explicitly for the externalities. In other words, the externality should have been internalized and not ignored. Hence, we show that the limiting policy is inefficient in this growth model with externalities. Moreover, the convergence of policies is monotonous, in the sense that the non-cooperative policy becomes increasingly inefficient as the number of sectors grows.

The rest of the paper is organized as follows: the next section discusses the general differential game with production; the third section specializes the general model of our specific formulation, the fourth section contains the main results of the paper, and the final section concludes.

\section{The General Problem}

Consider a world with only $\mathrm{n}$ industrial sectors, each endowed with a certain amount of labor per period (we normalize the endowment of labor to one in each industry). In period t industry $i, i=1 \ldots n$, owns stocks of capital $x_{i t}$. When this stock of capital is combined with sector-specific labor the industry obtains sector-specific effective labor. In this sense, capital amplifies the productivity of raw labor. Effective labor is used to produce consumption and capital goods. If industry $i$ assigns a fraction $l_{i t}$ of its labor time to the production of consumption goods, it obtains

$$
c_{i t}=h_{i}\left(l_{i t}, x_{i t}\right), \quad l_{i t} \in[0,1], i=1 \ldots n .
$$

There is an alternative use of labor time. In each sector, capital is created by investing a fraction of labor time. The production function for each type of capital is given by:

$$
x_{i t+1}=f\left(x_{i t}, l_{i t}, x_{-i t}, l_{-i t}\right), \quad i=1 \ldots n .
$$

where for any vector $v \in \Re^{n}$ we define $v_{-i} \equiv\left(v_{1}, \ldots v_{i-1}, v_{i+1}, \ldots v_{n}\right)$. Thus next period's stock of capital depends on (depreciated) capital plus the effective labor investment in capital. These production functions for capital exhibit an externality, because the accumulated stock of each type of capital influences the productivity of new investments (in terms of effective labor) in additional capital in sector $i$. There are various possible interpretations for this externality. If capital is interpreted as 
human capital, it might reflect the effect of a higher level of average education on the acquisition of additional education, or it might correspond to the notion that discoveries, inventions and ideas from one sector may be found useful in other areas. ${ }^{1}$

Each sector consumes the good it produces. We assume that industry 1 chooses the assignment of labor to consumption and investment by solving the following general dynamic programming problem:

$$
\begin{aligned}
V_{i}\left(x_{i t}, x_{-i t}\right)= & \max _{\left\{l_{i t}\right\}} \sum \delta_{i}^{t} U\left(c_{i t}\right) \\
\text { s.t. } \quad & c_{i t}=h_{i}\left(l_{i t}, x_{i t}\right) \\
& x_{i t+1}=f\left(x_{i t}, l_{i t}, x_{-i t}, l_{-i t}\right) \\
& l_{i t} \in[0,1]
\end{aligned}
$$

There are major difficulties in analyzing this problem, since in this general form it is not even known whether there is a solution (see Fischer and Mirman [3]). In order to obtain an understanding of the effects at play, in the next section we proceed to examine an example that can be solved explicitly, but which we believe captures most of the important features of the general model.

\section{The Main Example}

In this section we examine a particular specification of the production and utility functions. The following functional forms for utility and production allow for an explicit solution of the dynamic programming problems (3). Suppose production of the consumption good is

$$
c_{i t}=\left(1-l_{i t}\right) x_{i t}, \quad l_{i t} \in[0,1], i=1, \ldots n .
$$

while production of human capital is given by

$$
x_{i t+1}=\left(\rho x_{i t}+l_{i t} x_{i t}\right)^{\alpha}\left[\prod_{j \neq i}\left(\rho x_{j t}+l_{j t} x_{j t}\right)^{\beta /(n-1)}\right], \quad|\beta| \leq \alpha \leq 1, i=1 \ldots n .
$$

This is a natural extension of the "fish production" models of Fischer and Mirman [3] to the case of a symmetric model of many interacting sectors in a standard production economy. ${ }^{2}$ In the production function, $\rho x_{i t}$ corresponds to depreciated capital and $l_{i t} x_{i t}$ is the investment in capital stock in period $t$ in sector $i$. The external effects caused by the capital stocks of the other sectors act through the term in brackets.

Using Bellman's principle for the value function (3) and the specific functional forms above, we can rewrite the value function to obtain the maximization problem facing each sector: 


$$
\begin{aligned}
V\left(x_{i t}, X_{-i t}\right)= & \max _{\left\{l_{i t}\right\}} \log c_{i t}+\delta V\left(x_{i t+1}, X_{-i t+1}\right) \\
\text { s.t. } & x_{i t+1}=\left(\rho x_{i t}+l_{i t} x_{i t}\right)^{\alpha}\left[\prod_{j \neq i}\left(\rho x_{j t}+l_{j t} x_{j t}\right)^{\beta /(n-1)}\right] \\
& c_{i t}=\left(1-l_{i t}\right) x_{i t} \\
& 0 \leq l_{i t} \leq 1, \quad i=1, \ldots, n .
\end{aligned}
$$

In what follows, we assume that these value functions are finite. ${ }^{3}$

\section{Noncooperation}

In this section we follow the methodology of Fischer and Mirman [3] to obtain an explicit solution to the optimization problem of each industry and use it to show that as the number of sectors grows, the solution approaches the optimal solution when there are no externalities. Before proving our main result, we obtain an explicit solution to the $n$ sector model in the following lemma:

Lemma 1 A symmetric, stationary solution to the dynamic game described by (6) is given by the linear rule $c_{i}=\gamma x_{i}$, where ${ }^{4}$

$$
\gamma=\frac{(1+\rho)(1-\delta \alpha-\delta \beta)(1-\delta \alpha-\delta \beta-n(1-\delta \alpha))}{(1-\delta \alpha-2 \delta \beta)-n(1-\delta \alpha-\delta \beta)}
$$

Proof: Following Fischer and Mirman[3], we assume a solution for sector $i$ of the form:

$$
V_{i}\left(x_{i}, X_{-i}\right)=A_{i} \log x_{i}+\sum_{j \neq i} B_{i j} \log x_{j}+D_{i}
$$

and that $c_{i t}=\gamma_{i} x_{i t}, i=1, \ldots, n .^{5}$ By Bellman's equation, we can write

$$
\begin{aligned}
& A_{i} \log x_{i}+\sum_{j \neq i} B_{i j} \log x_{j}+D_{i}= \\
& =\log c_{i}+\delta A_{i} \log \left\{\left(\rho+1-\gamma_{i}\right) x_{i}^{\alpha} \prod_{j \neq i}\left[\left(\rho+1-\gamma_{j}\right) x_{j}\right]^{\beta /(n-1)}\right\} \\
& +\delta \sum_{j \neq i}\left(B_{i j} \log \left\{\left(\rho+1-\gamma_{j}\right) x_{j}^{\alpha} \prod_{k \neq j}\left[\left(\rho+1-\gamma_{k}\right) x_{k}\right]^{\beta /(n-1)}\right\}\right)+\delta D_{i}
\end{aligned}
$$


Identifying constants and using the symmetry of the model, we obtain the equations:

$$
\begin{aligned}
A_{i} & =1+\delta \alpha A_{i}+\delta \sum_{j \neq i} B_{i j} \beta /(n-1), \quad i=1, \ldots, n \\
B_{i j} & =\delta\left[A_{i} \beta /(n-1)+\alpha B_{i j}+\beta /(n-1) \sum_{k \neq j, i} B_{i k}\right], \quad i \neq j=1, \ldots, n .
\end{aligned}
$$

Noting that under symmetry $A_{i}=A, B_{i j}=B$, we solve for $A$ and $B$ to obtain:

$$
\begin{aligned}
& A=\frac{(n-1)(1-\delta \alpha-\delta \beta)+\delta \beta}{(1-\delta \alpha-\delta \beta)((n-1)(1-\delta \alpha)+\delta \beta)} \\
& B=\frac{\delta \beta}{(1-\delta \alpha-\delta \beta)((n-1)(1-\delta \alpha)+\delta \beta)}
\end{aligned}
$$

Maximizing (6) with respect to $\gamma_{i} \equiv \gamma, i=1, \ldots, n$ we obtain the first order conditions:

$$
\frac{1}{\gamma}=\delta\left[\frac{A \alpha+\beta B}{\rho+1-\gamma}\right]
$$

from which we obtain the value for $\gamma$ given in (7) by replacing the value of $A$ and $B$ in the previous expression and solving.

For example, in the case $n=2$, we recover a solution that is analogous to the one obtained by Fischer and Mirman [3], for a production technology:

$$
\gamma_{i}=(1+\rho)\left((1-\delta \alpha)-\frac{\delta^{2} \beta^{2}}{1-\delta \alpha}\right), \quad i=1,2
$$

We use this lemma to prove the main result of the paper.

Proposition 1 A symmetric, stationary solution to the dynamic game described by (6) exists and as the number of sectors increases, the fraction of labor allocated to consumption approaches the optimal (cooperative) solution when there are no externalities,

$$
\gamma_{i}=(1+\rho)(1-\delta \alpha), \quad i=1 \ldots n .
$$

Proof: Taking the limit as $n \rightarrow \infty$ in the expression for $\gamma$ obtained in (7) we get the desired result.

The first thing to note about this result is that the consumption rule given in the proposition is analogous to the one obtained by Levhari and Mirman [4] for the cooperative -i.e., optimal- solution in a case of no externality. That solution is not efficient here, since it does not consider the existence of the production externality. 
This proposition extends to a dynamic game the results that obtain in a one shot game with external effects: as the number of sectors grows with no bounds, we arrive at the problem of the commons.

It is interesting to compare the solution to the non-cooperative problem with the cooperative solution obtained when the planner maximizes collective welfare. Using the same techniques as those used to derive proposition 1, it is easy to show that $\gamma^{c}$, the proportion of raw labor that is devoted to consumption is: ${ }^{6}$

$$
\gamma^{c}=(1+\rho)(1-\delta \alpha-\delta \beta)
$$

If we compare the cooperative to the non-cooperative solution as the number of sectors increases, we have that the difference is:

$$
\gamma_{i}^{c}-\gamma_{i}=(1+\rho) \frac{(1-\delta \alpha-\delta \beta)(n-1) \delta \beta}{(1-\delta \alpha-2 \delta \beta)-n(1-\delta \alpha-\delta \beta)}
$$

and we have $\operatorname{sgn}\left(\gamma_{i}^{c}-\gamma_{i}\right)=-\operatorname{sgn}(\beta)$. Moreover, the extent of the inefficiency increases with the number of sectors, $n$. In the limit when $n \rightarrow \infty,\left(\gamma_{i}^{c}-\gamma_{i}\right)=$ $-\delta \beta(1+\rho)$. What these expressions are telling us is that if the externalities are positive, as in the case of knowledge spillovers between sectors, the non-cooperative consumption rate is too high. On the other hand, if the externality is negative, as in the case of pollution between sectors, the non-cooperative consumption rate is too low. Moreover, the convergence to the limiting policy is monotonous, in the sense of more inefficiency as the number of sectors increases.

\section{Conclusions}

This paper has examined the dynamics of growth in a production model with $n$ sectors that generate externalities that affect the other sectors. We have found a solution to the dynamic non-cooperative game between the different industrial sectors. The comparison between this solution and the optimal cooperative solution shows that the allocation of resources to investment is inefficient and that the inefficiency is due to lack (excess) of investment when the interactions are positive (negative).

The main result of this paper is derived from an examination of the solution of the game as the number of sectors increases. We find the limiting policy, which replicates the optimal policy when there are no production externalities. The limiting policy is hence inefficient and the over- (or under-) consumption which is the source of the inefficiency depends on the sign of the externality between sectors. In other words, the behavior of each sector approaches the behavior of an industry which has no (or which is not aware of) interactions with other industries, i.e., the usual assumption in endogenous growth models.

It is interesting to note that the model can also be interpreted as a world of many countries whose economies interact only through externalities such as crossboundary pollution or through the diffusion of knowledge. 


\section{Acknowledgments}

We have benefitted from helpful discussions with Manjira Datta. We also wish to thank an anonymous referee. This paper was made possible by project FONDECYT 1940507. Ronald Fischer would like to thank the Department of Economics of the University of Pennsylvania for its hospitality during a crucial part of the writing of this paper.

\section{Notes}

1. The model includes the possibility of negative interactions. If the stock is believed to be physical capital, pollution or other environmental effects would lead to negative interactions. An alternative interpretation of the model considers $n$ countries, with one industry per country. In this case a negative externality would reflect cross-boundary pollution.

2. Somewhat more generally, we could have used different external effects, i.e., $\beta_{i} \neq \beta_{j}$, with $\sum\left|\beta_{i}\right| \leq \alpha$. We would have lost the symmetry of the present model which admits a simple solution, without a large gain in terms of generality.

3. See, for instance, the appendix of Fischer[2].

4. See Fischer and Mirman [3] for arguments that support (without proving) the uniqueness of the solution.

5. We omit the calculation of $D_{i}$, since it is not used in the sequel.

6. This result is analogous to the one obtained in Fischer and Mirman [3], for a production economy.

\section{References}

1. P. Romer. Increasing Returns and Long Run Growth, Journal of Political Economy, 94:100237, 1986.

2. R. Fischer. Capital Movements in a World with Production Externalities, International Economics Research Unit \# 34, University of Pennsylvania, 1995.

3. R. Fischer and L. Mirman. Strategic Dynamic Interactions: Fish Wars, Journal of Economic Dynamics and Control, 16:167-87, 1992.

4. D. Levhari and L. Mirman. The Great Fish War: An Example Using a Dynamic CournotNash Solution, Bell Journal of Economics, 11:322-34, 1980 


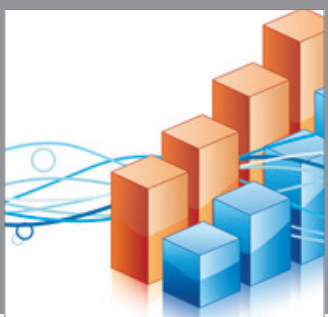

Advances in

Operations Research

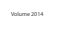

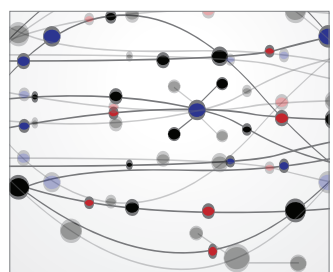

\section{The Scientific} World Journal
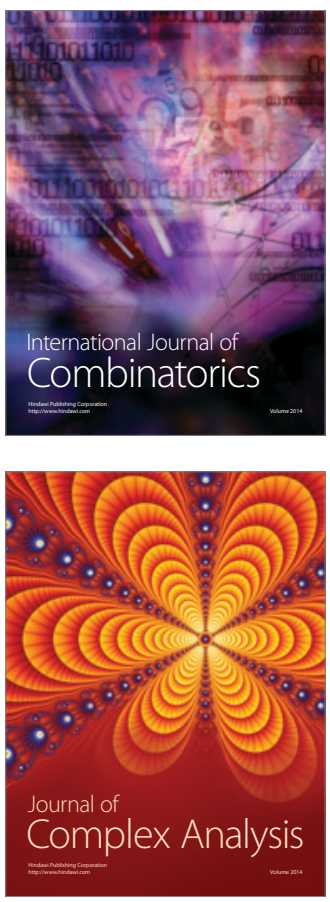

International Journal of

Mathematics and

Mathematical

Sciences
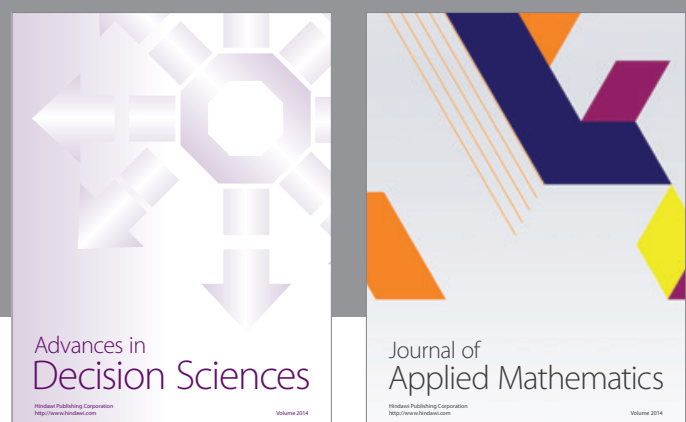

Journal of

Applied Mathematics
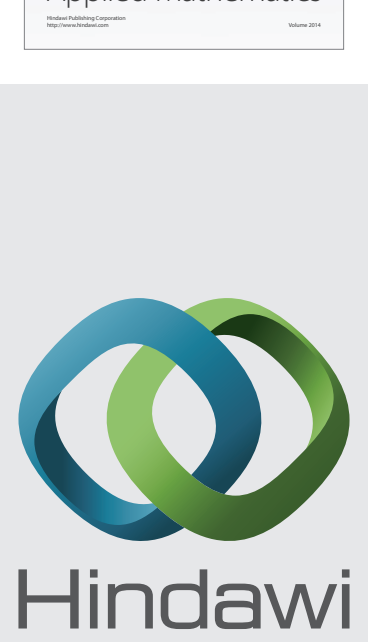

Submit your manuscripts at http://www.hindawi.com
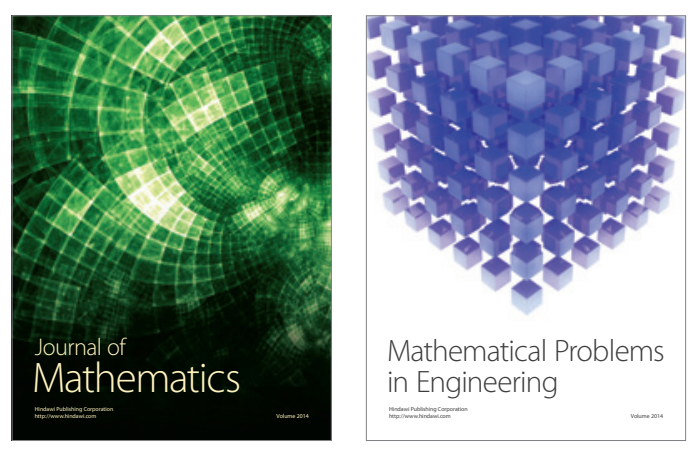

Mathematical Problems in Engineering
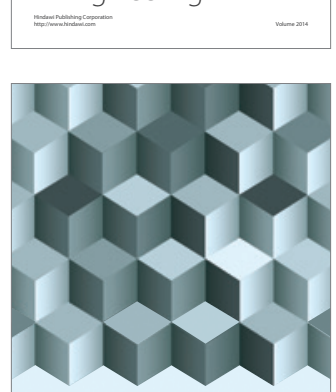

Journal of

Function Spaces
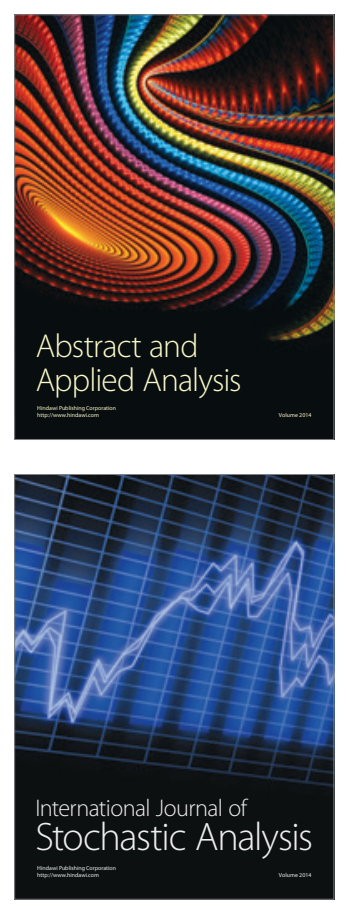

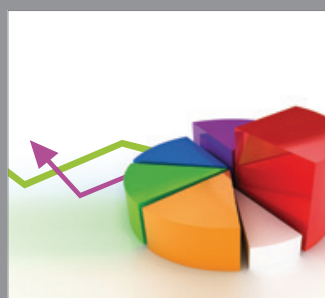

ournal of

Probability and Statistics

Promensencen
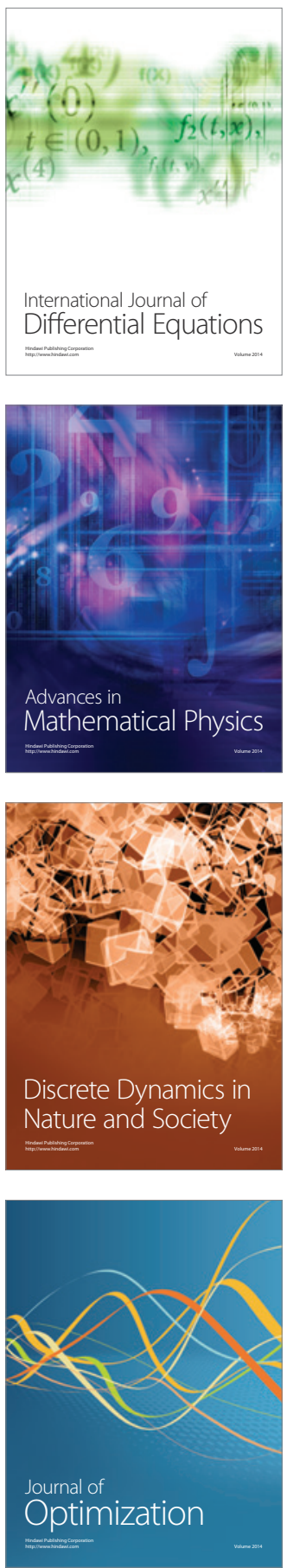